\title{
United airway disease: current perspectives
}

This article was published in the following Dove Press journal:

Journal of Asthma and Allergy

II May 2016

Number of times this article has been viewed

\section{Pedro Giavina-Bianchi* \\ Marcelo Vivolo Aun* \\ Priscila Takejima \\ Jorge Kalil \\ Rosana Câmara Agondi}

Clinical Immunology and Allergy

Division, Faculty of Medicine,

University of São Paulo, São Paulo,

Brazil

*These authors contributed equally to this work.
Correspondence: Pedro Giavina-Bianchi Clinical Immunology and Allergy Division, Faculty of Medicine, University of São Paulo, I78 Rua Professor Artur Ramos apartment 2I I A, Jardim América, São Paulo 0I454-904, Brazil

Tel/fax +55 I I 307। 3 I89

Email pbianchi@usp.br

\begin{abstract}
Upper and lower airways are considered a unified morphological and functional unit, and the connection existing between them has been observed for many years, both in health and in disease. There is strong epidemiologic, pathophysiologic, and clinical evidence supporting an integrated view of rhinitis and asthma: united airway disease in the present review. The term "united airway disease" is opportune, because rhinitis and asthma are chronic inflammatory diseases of the upper and lower airways, which can be induced by allergic or nonallergic reproducible mechanisms, and present several phenotypes. Management of rhinitis and asthma must be jointly carried out, leading to better control of both diseases, and the lessons of the Allergic Rhinitis and Its Impact on Asthma initiative cannot be forgotten.
\end{abstract}

Keywords: ARIA, united airway disease, rhinitis, asthma, allergy, atopy, immediate hypersensitivity reaction, guideline

\section{Introduction}

Upper and lower airways are considered a unified morphological and functional unit, and the connection existing between them has been observed for many years, both in health and in disease. ${ }^{1,2}$ More than 2,000 years ago, Claudius Galenus studied the upper airway and paranasal sinuses as integral parts of the respiratory tract, and he assumed that rhinitis and asthma were caused by secretions dripping from the brain into the nose and lung. ${ }^{3}$ More recently, the concept of united airway disease (UAD) was suggested. ${ }^{4-6}$

The nose is situated at the entrance of the airway and protects the lower airway from the harmful effects of the inspired air by acting as efficient air-conditioning. The nose warms, filters, and humidifies the inspired air so that clean air that is fully saturated with water vapor at a temperature of $37^{\circ} \mathrm{C}$ is delivered to the lungs. During nose breathing, the majority of particles with an aerodynamic equivalent diameter (AED) $>15 \mu \mathrm{m}$ are deposited in the upper respiratory tract. Particles with AEDs $>2.5 \mu \mathrm{m}$ are primarily deposited in the trachea and bronchi, whereas those with lower AEDs penetrate into the gas-exchange region of the lungs. ${ }^{7}$ The nasal and bronchial mucosa present similarities, and one of the most important concepts regarding nose-lung interactions is the functional complementarity, which assigns the protector role of the nose to the lungs. ${ }^{4}$ However, the functions of the upper airway and their interactions with the lower airway are much broader than merely air-conditioning.

The Allergic Rhinitis and Its Impact on Asthma (ARIA) guidelines published in $2001^{1}$ achieved some goals: 1 ) development of a guideline proposing a standardized 
management plan for allergic rhinitis (AR), 2) establishment of the ARIA concept, 3) spreading of the guideline to general and specialist physicians, and 4) establishment of a multiprofessional forum to study rhinitis and asthma. There is strong epidemiologic, pathophysiologic, and clinical evidence supporting an integrated view of rhinitis and asthma: UAD in the present review. We can also consider UAD an airway-hypersensitivity syndrome, because rhinitis and asthma are chronic inflammatory diseases of the upper and lower airways, which are induced and reproduced by allergic or nonallergic hypersensitivity reactions, and present several phenotypes (Table 1).

\section{United airway disease: epidemiologic evidence}

AR is the most common of all atopic diseases, and although it can develop at any age, most patients report the onset of symptoms before 30 years of age, making it the most common chronic disorder in children. ${ }^{6}$ AR can be considered a major public health problem, due to its prevalence and impact on patients' quality of life, work/school performance, and productivity economic burden., ${ }^{4,6}$ It is characterized by the classic symptoms of nasal itching, sneezing, rhinorrhea, and nasal obstruction. In addition, AR is associated with a variety of comorbidities, such as atopic dermatitis, sleepdisordered breathing, conjunctivitis, rhinosinusitis, otitis media, asthma, and emotional problems. ${ }^{6,8}$ At the same time, $\mathrm{AR}$ is a disease that is underdiagnosed and overlooked by patients and physicians. ${ }^{9,10}$

AR is considered a risk factor for developing asthma. ${ }^{4,6}$ Asthma is a heterogeneous disease characterized by chronic airway inflammation and hyperresponsiveness (AHR) to direct or indirect stimuli, which can persist even when symptoms are absent or lung function is normal but may normalize with treatment. ${ }^{11}$ Asthma is defined by the history of episodic respiratory symptoms, such as wheeze, shortness of breath, chest tightness, and cough, and is associated with variable expiratory airflow limitation. ${ }^{11}$

Allergic asthma is the most prevalent disease phenotype, which often begins in childhood and is associated with a personal and/or family history of allergic diseases, such as

Table I Airway hypersensitivity syndrome phenotypes

I. Rhinitis ${ }^{\mathrm{a}}$
2. Rhinitis ${ }^{\mathrm{a}}$ with airway hyperresponsiveness
3. Eosinophilic bronchitis
4. Asthma
5. Rhinitis ${ }^{\mathrm{a}}$ and asthma

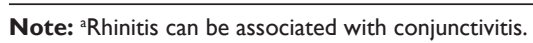

eczema and AR. ${ }^{11}$ In the same way of the allergic phenotype, patients with non-AR (NAR) are at increased risk of developing nonallergic asthma. NAR presents later in life than AR and is not a single disorder but is composed of a heterogeneous group of diseases. ${ }^{12}$

According to the International Study on Asthma and Allergy in Childhood, the prevalence of AR in Europe was found to be $\sim 25 \%$ and in Brazil $\sim 15 \%-20 \%$. The prevalence of asthma worldwide was observed to be $\sim 20 \%$ (Global Initiative for Asthma) and 10\%-20\% in Brazil (Global Initiative for Asthma, ARIA). Countries with a very high prevalence of rhinitis had asthma prevalence ranging from $10 \%$ to $25 \%$. $^{4,11,13}$

The management of asthma should include assessment of asthma control, future risks, and any comorbidity that could contribute to symptom burden and poor quality of life. The main associated comorbidities are rhinitis, rhinosinusitis, gastroesophageal reflux, obesity, obstructive sleep apnea, depression, and anxiety. ${ }^{11}$ We evaluated the prevalence of comorbidities in patients with severe asthma and observed that rhinitis and gastroesophageal reflux disease were the most common, rhinitis being observed in $91 \%$ and gastroesophageal reflux disease in $71 \%$ of the asthmatic patients. ${ }^{14}$

Interactions between the lower and the upper airways are well known and have been extensively studied since 1990 . Over $80 \%$ of asthmatics have rhinitis, and $10 \%-40 \%$ of patients with rhinitis have asthma, suggesting the concept of "one airway, one disease". ${ }^{4}$ Rhinitis symptoms have been reported in $98.9 \%$ of allergic asthmatics and in $78.4 \%$ of nonallergic asthmatics. Furthermore, $-30 \%$ of patients with only AR who do not have asthma present hyperresponsiveness to methacholine or histamine. ${ }^{2,4,15}$ However, there are large differences in the magnitude of airway reactivity between patients with rhinitis and asthma. Patients with perennial rhinitis have greater bronchial reactivity than those with seasonal rhinitis, in whom the presence of hyperresponsiveness was observed especially during the pollen season. ${ }^{4,15,16}$ AHR, which is a paramount feature of asthma, is a strong risk factor for the onset of asthma in patients presenting with AR. ${ }^{6}$

Several studies suggest that AR and NAR are risk factors for new onset of asthma and persistence of asthma. ${ }^{17,18}$ In a cohort of 690 individuals with a follow-up of 23 years, it was observed that the incidence of asthma was $10.5 \%$ in subjects with rhinitis and $3.6 \%$ in those without rhinitis. Therefore, the development of asthma was tripled in rhinitis patients compared to those without rhinitis. ${ }^{19}$ In the Tucson Epidemiologic Study of Obstructive Lung Diseases, the odds ratio for developing asthma was 2.59 (95\% confidence 
interval 1.54-4.34) if rhinitis was present and 6.28 (95\% confidence interval 4.01-9.82) in the presence of rhinitis plus sinusitis..$^{20}$ A European Survey confirmed the presence of perennial rhinitis as a major risk factor for asthma, with odds ratios of 11 for the atopic and 17 for the nonatopic phenotype. ${ }^{21}$

Asthma and rhinitis share common risk factors and present common susceptibility to different agents, such as allergens (atopy) and infections. ${ }^{4,6}$ The presence of AHR and concomitant atopic manifestations in childhood increases the risk of developing asthma and should be recognized as a marker of prognostic significance, whereas the absence of these manifestations predicts a very low risk of future asthma. ${ }^{22}$

\section{United airway disease: pathophysiological evidence}

The upper and lower respiratory tracts form a continuum, allowing the passage of air into and out of the lungs and sharing many anatomical and histological properties. ${ }^{2}$ They share common structures, including the ciliary epithelium, basement membrane, lamina propria, glands, and goblet cells, forming the so-called united airway. ${ }^{23}$ On the other hand, differences between the upper and lower airways do exist. Nasal mucosa, which is attached to bone, is enriched with vessels, whereas bronchial mucosa, which is attached to cartilage, is enriched with smooth-muscle cells. ${ }^{24}$ Therefore, the major cause of airway obstruction, especially in the early phase of the allergic response, is different: upper airway obstruction is caused by vasodilation and edema, whereas lower airway obstruction arises from smooth-muscle constriction. ${ }^{24}$

It is reasonable to think that because of anatomic reasons, the upper airway constitutes the first target for allergens and for physical and chemical environmental stimuli; therefore, they tend to be the first to be affected by the allergic airway disease, and if the intensity of this disease is low, the upper airway may be the only part of the respiratory tract that is affected. However, when the entire respiratory tract is involved, rhinosinusitis and asthma follow a parallel course. ${ }^{25}$ Unfortunately, systematic research in this field has not been performed, and the evidence supporting these postulates is scarce. ${ }^{25}$

UAD presents two main phenotypes: allergic (atopic or extrinsic) and nonallergic (nonatopic or intrinsic). With regard to asthma, most children and at least $50 \%$ of adults have the allergic phenotype, in which the disease is associated with allergic sensitization defined by the presence of serum-specific immunoglobulin (Ig)E antibodies and/or positive skin tests to the proteins of common inhaled allergens, such as house dust mites, animal dander, fungal spores, pollens, and cochroaches. ${ }^{26}$ On the other hand, in nonallergic asthma, we do not observe IgE reactivity to allergens. ${ }^{26}$ In the same way, there are two important phenotypes or rhinitis, allergic and nonallergic, both of them associated with increased prevalence of asthma. ${ }^{27} \mathrm{We}$ focus on the allergic pathophysiology of UAD.

$\mathrm{AR}$ and atopic asthma result from an IgE-mediated allergic reaction associated with airway inflammation of variable intensity. ${ }^{4}$ Since the first class of Ig presented on the surface of B-cells is $\operatorname{IgM}$, it is necessary that $\operatorname{IgM}$ is switched to $\operatorname{IgE}$ so that allergic inflammation can develop. Isotype switching to IgE requires antigen presentation and two other signals..$^{28}$ Signal one is provided by interleukin (IL)-4 and/or IL-13, acting through IL-4R and IL-13R via STAT6, which activate transcription to the $\operatorname{IgE}$ isotype. Signal two is provided mainly by ligation of CD40 on B-cells to CD40L on T-cells, which activates DNA-switch recombination. ${ }^{28}$ The IgE-mediated immune response is initiated when the allergens are taken up by antigen-presenting cells via the cell-surface Ig receptor. Processed fragments are then presented in the context of major histocompatibility complex class II to T-helper $\left(\mathrm{T}_{\mathrm{H}}\right)$ cells, which recognize the allergen-major histocompatibility complex II composite and are activated. The allergen-specific $\mathrm{T}_{\mathrm{H}} 2$ cells produce IL- 4 and IL-13, and express CD154, leading to IgE class switching. ${ }^{26,28}$ Although class switching is generally thought to occur in the germinal center of lymphoid tissues, it has also been reported to occur in the respiratory mucosa of patients with AR and atopic asthma and in the gastrointestinal tract in patients with food allergy. ${ }^{28}$

Once IgE is produced by B-cells, the Ig will bind to the high-affinity receptor $\mathrm{Fc}_{\varepsilon} \mathrm{R} 1$ on mast cells and basophils. ${ }^{28}$ In future, contacts with the polyvalent sensitizing allergen, these cells will be activated through $\mathrm{Fc}_{\varepsilon} \mathrm{R} 1$, initiating an immediate hypersensitivity reaction that is central in the pathogenesis of AR and allergic asthma. ${ }^{28}$ The reaction has an immediate phase that is induced by the release of preformed and rapidly synthesized mediators from mast cells and basophils, resulting in erythema, edema, and itching in the skin, sneezing and rhinorrhea in the upper respiratory tract, and cough, bronchospasm, edema, and mucous secretion in the lower respiratory tract. ${ }^{28} \mathrm{~A}$ late phase mediated by cytokines and chemokines and characterized by edema and leukocytic influx can occur 6-24 hours after the immediate phase. Eosinophils recruited mainly by IL-5 produced by $\mathrm{T}_{\mathrm{H}} 2$ cells stand out and are essential to maintain the chronic inflammatory process and tissue damage. ${ }^{28}$

Eosinophil activation directly contributes to vasodilation, edema, mucous production, bronchoconstriction, and 
dysfunctional remodeling of the airway. ${ }^{29}$ These processes are mainly induced by eosinophil-derived products, such as eosinophil peroxidase, which causes AHR and activates dendritic cells. ${ }^{26}$ Murine studies have shown that eosinophils also contribute to airway-wall remodeling and subepithelial membrane thickening via the release of TGF $\beta .{ }^{26}$ Finally, similarly to neutrophils, upon activation, eosinophils undergo cytolysis and release mediators from the eosinophilic granules, such as eosinophil-derived neurotoxin, cationic proteins (eosinophil peroxidase), and major basic protein, which can damage structural cells of the airway. ${ }^{26}$ It has been demonstrated that humans who died from asthma presented eosinophilic inflammation all over the respiratory tract, from nasal mucosa to lung tissue, showing that the airways really are unique, even in pathologic conditions. ${ }^{30}$ Therefore, AR and asthma share immunopathological features, including a $\mathrm{T}_{\mathrm{H}}$ 2-type immune response, thickness of the basement membrane, and goblet-cell hyperplasia. ${ }^{24}$

In contrast to allergic UAD, the pathophysiology of which is well characterized, the etiology of and mechanisms involved in nonallergic UAD remain unclear. Some of the possibilities include allergy triggered by unknown antigens (fungi), persistent infection (caused by Chlamydia trachomatis, Mycoplasma spp., or viruses), and autoimmunity.

A central concept of UAD is the influence of the upper airway in the function of the lower airway, which is particularly evident and relevant in the allergic phenotype. ${ }^{25}$ The pathological interactions between the upper and lower airways are summarized in Figure 1 and can be divided into:

- air-conditioning

- inflammation

- neural reflexes.

\section{Air-conditioning}

Galen was the first to offer insights on the function of the nose as protector of the lower airway through its ability to clean, warm, and humidify inhaled air. ${ }^{5}$ In addition, the nasal mucosa, with its abundant submucosal glands, takes part of the innate and adaptive immune defense by releasing antibacterial proteins, such as lysozyme and lactoferrin, chemical defenses, antioxidants, and secretory $\operatorname{IgA}$, that can protect the lower airway from pathogens and allergens. ${ }^{25}$ Patients with AR present partial or complete loss of function of the nose due to mucosal congestion, since nasal airways are bypassed during oral breathing. ${ }^{25}$ In this situation, inhalation of cold and dry air may directly induce bronchoconstriction. Therefore, the lower airway would be quite "opened" to the entrance of allergens and pathogens, increasing the risk of asthma exacerbation.

\section{Inflammation}

Propagation of inflammation from the upper airway to lower airway may occur via postnasal drip and systemic circulation.

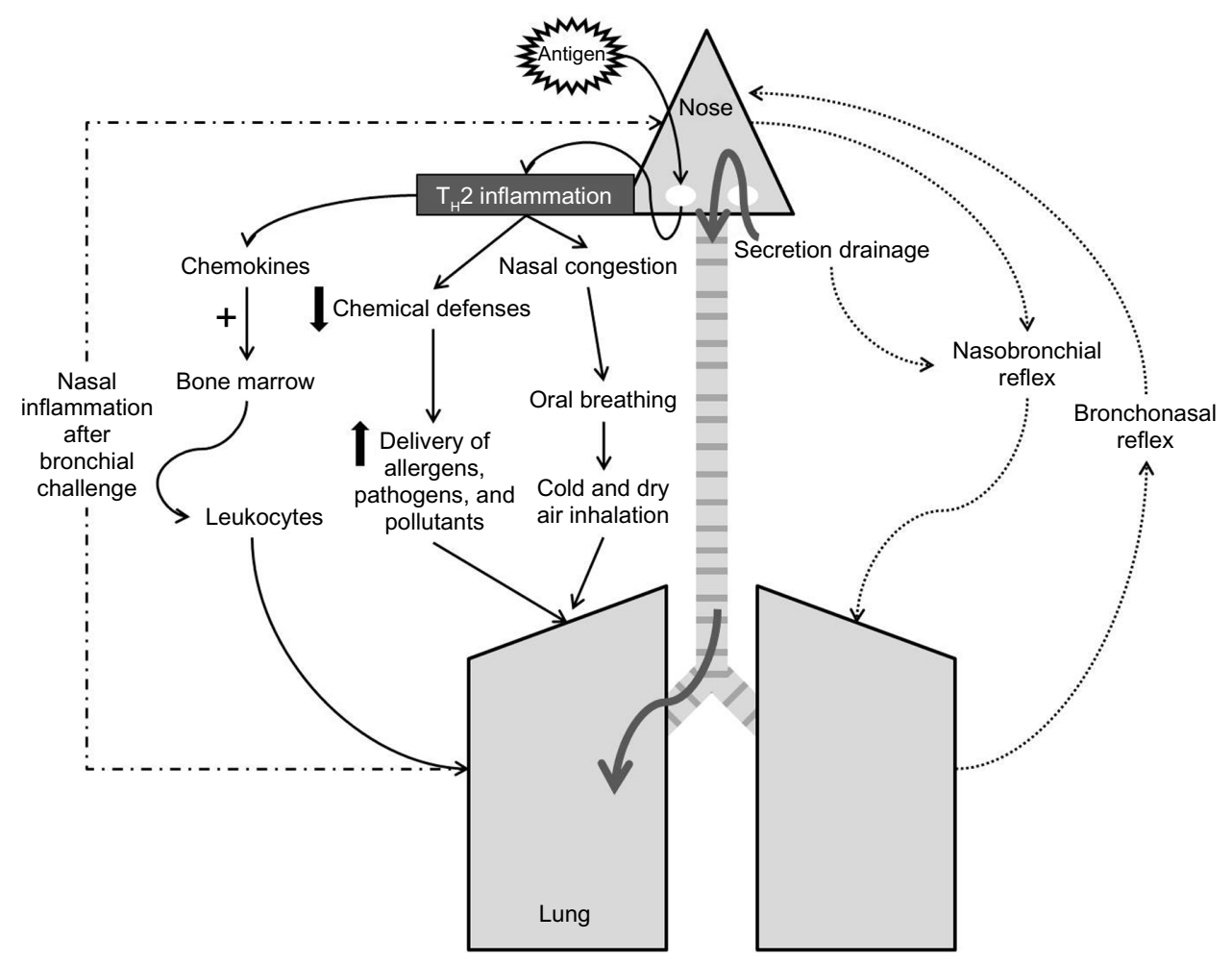

Figure I United airway disease: pathophysiological interaction. 
The concept that inflammatory secretions from the upper airway of patients with rhinosinusitis or even with rhinitis are aspirated into the lower airway with adverse consequences has been viewed as one of the principal mechanisms for lower airway symptoms, especially after an upper respiratory infection. ${ }^{25}$ It is quite possible that early morning coughing in individuals with rhinitis is associated with accumulation of secretions in the lower pharyngeal area stimulating irritant receptors. ${ }^{25}$ It is questionable, however, whether these secretions can reach the intrathoracic lower airway in adequate quantities to alter their physiology and to generate exacerbations or chronically worsen lower airway function in patients with asthma. ${ }^{25}$ The development of sinusitis in rabbits is associated with lower AHR, even after eliminating upper-lower airway communication with the use of an inflated endotracheal tube cuff. ${ }^{31}$

On the other hand, there is good evidence that allergic inflammation developing in the respiratory mucosa may result in systemic inflammatory events. ${ }^{25}$ Blood eosinophilia may be observed in patients with allergic asthma, and can be considered a biomarker of inflammation of the lower airway. There is less evidence that upper airway inflammation can lead to an increase in eosinophil blood count. ${ }^{25,32}$ Moreover, there is no experimental information indicating that nasal inflammation leads to systemic inflammatory signals that induce changes in lower airway physiology, ${ }^{25}$ even though it seems reasonable to speculate that cytokines released in nasal mucosa could activate bone marrow with chemotaxis of white blood cells to both upper and lower airways. ${ }^{25}$

The opposite direction for propagation of the inflammatory process, beginning in the lower airway and getting to the upper airway, has been postulated. A study showed that segmental bronchial allergen provocation in patients with nonasthmatic AR can induce nasal inflammation, nasal and bronchial symptoms, and reduction in pulmonary and nasal function. ${ }^{33}$ However, there are recent data suggesting that this lung-nasal propagation of inflammation might not be relevant. In a very elegant murine model of allergic respiratory inflammation induced by ovalbumin, Balb/c mice were submitted to intratracheal challenge after sensitization by an intraperitoneal route. This provocation induced lung inflammation and AHR, but no signs of inflammation were found in the nose. ${ }^{34}$

\section{Neural reflexes}

The existence of a nasobronchial reflex that originates from the sensory nerve endings in the nose, travels to the central nervous system through the trigeminal nerve, and follows an efferent pathway through the vagus nerve to produce airway smooth-muscle contraction has been under debate for years. ${ }^{25}$ Despite being well documented in animal models, its existence and relevance in humans are still controversial.

Some studies performed in healthy individuals and asthmatics have demonstrated that lower airway resistance increased after nasal inhalation of cold and dry air. ${ }^{35,36}$ Another important study showed an increase in AHR after a nasal allergen provocation in asthmatics who had reported worsening of asthma symptoms following seasonal exacerbations of rhinitis. ${ }^{37}$ The authors observed that none of the solutions delivered to the nose during allergen provocation could be detected in the lower airway, showing that the increase in AHR was not due to inadvertent inhalation of the allergen. ${ }^{37}$ It is important to point out that the classic nasobronchial reflex is a component of the diving reflex. ${ }^{38}$ Immersion of the head into cold water leads to immediate suppression of respiration (apnea), laryngospasm, and bronchoconstriction, in order to protect the lower airway from diving. ${ }^{38}$ Nasal inhalation of dust, pollutants, and irritants can induce immediate bronchoconstriction with cessation of respiration in the expiratory phase, due to relaxation of inspiratory muscles. ${ }^{38}$ Therefore, in individuals with allergic respiratory disease, this reflex could lead to an increase in asthma symptoms after nasal injury.

There is less evidence showing the occurrence of a bronchonasal reflex. It has been demonstrated that inhalation of ultrasonically nebulized distilled water increased nasal airway resistance in patients with $\mathrm{AR}$, without the involvement of parasympathetic efferent reflexes, since patients did not present sneezing or rhinorrhea. ${ }^{39}$ The clinical relevance of this bronchonasal reflex has yet to be demonstrated.

In conclusion, upper and lower airways seem to constitute a unique system, named "united airway", that share similarities in terms of histology, physiology, and pathology. UAD is triggered by a $\mathrm{T}_{\mathrm{H}} 2$ immune response of the airway, leading to an extended inflammatory process that begins in nasal mucosa and ends in bronchioles and alveoli, particularly in symptomatic asthmatics.

\section{United airway disease: clinical evidence}

There is also clinical evidence supporting the concept of UAD. Studies have demonstrated that the presence of severe rhinitis is associated with an increased risk of asthma ${ }^{20}$ and in patients with asthma a less favorable evolution. ${ }^{40-42}$ It has also been shown that the treatment of rhinitis can be beneficial to the lower airway, reducing symptoms, emergency room visits, and hospitalizations, as well as the severity of bronchial hyperresponsiveness. ${ }^{43-47}$ In protocols of difficult-to-control 
asthma, rhinitis was included as one of the main comorbidities to be assessed and treated. ${ }^{48}$

Therapy for UAD includes avoidance of relevant allergens and irritants, pharmacotherapy, and allergen-specific immunotherapy (SIT). Allergen avoidance has been suggested not only to prevent UAD onset and progression but also to reduce its burden, improving symptoms and quality of life. However, there is a lack of evidence supporting the effectiveness of environmental control. ${ }^{4}$

The pharmacologic approach of AR includes antihistamines, oral leukotriene antagonists, and intranasal corticosteroids, the last being considered the most efficacious drug. ${ }^{4}$ Agondi et al reported a decrease in asthma symptoms and AHR after intranasal corticosteroid treatment of rhinitis. ${ }^{43}$ A recent meta-analysis confirmed the beneficial effect of intranasal steroids in AHR. ${ }^{44}$ Oral and intranasal antihistamines, as well as leukotriene antagonists, are less effective than intranasal corticosteroids in improving the symptoms of AR. ${ }^{49-52}$ A protective effect of cetirizine against AHR measured 6 hours after nasal allergen challenge in patients with AR was shown. ${ }^{53}$

Allergen-SIT is defined as a procedure to administer increasing amounts of specific allergens in patients diagnosed with IgE-mediated disease, in order to induce immune tolerance. ${ }^{8,54}$ Subcutaneous and sublingual IT can reduce symptoms of AR and need of reliever medication, as well as improve the control of comorbid conditions, such as asthma and conjunctivitis. ${ }^{8,55}$

Allergen-SIT is indicated in moderate/severe AR for which response to pharmacotherapy is inadequate. Other potential indications are adverse effects of medications, coexisting allergic asthma, bad adherence to therapy, and patient preference for IT instead of pharmacotherapy. ${ }^{8,56}$ Furthermore, SIT has been positioned as the only treatment that can modify the natural course of allergic diseases that includes prevention of new sensitizations and reduction of risk of developing asthma in subjects with AR, even after termination of treatment. ${ }^{57-61}$ The Preventive Allergy Treatment study was a randomized controlled trial that showed clinical benefits and a preventive effect on asthma development in children suffering from seasonal rhinoconjuctivitis undergoing subcutaneous IT with grass- and/or birch-allergen extracts for 3 years. This positive effect of SIT in preventing the progression from rhinitis to asthma was observed to persist in the same patients for 7 years after the termination of the treatment. ${ }^{57-59}$ Another study found that after 3 years of sublingual grass-pollen IT in children with AR, eight of 45 actively treated subjects and 18 of 44 controls developed asthma, with 3.8-fold more frequent development of asthma in the untreated patients. ${ }^{62}$

Some studies have shown that IT was able to prevent new sensitizations in monosensitized individuals. ${ }^{63} \mathrm{~A}$ research assessing the effects of subcutaneous IT in 147 house dust mite-monosensitized children over 5 years found similar results: $75.3 \%$ in the treated group and $46.7 \%$ in the control group had no new sensitizations. ${ }^{64} \mathrm{~A}$ randomized controlled study involved 216 children with AR (with or without intermittent asthma) receiving drugs alone or drugs plus sublingual IT for 3 years showed new sensitizations in $34.8 \%$ of controls and in $3.1 \%$ of the IT group. Moreover, they demonstrated that this protective effect extended to AHR, which significantly decreased in the IT group. ${ }^{65}$

Novel targeted therapeutic approaches using biological agents have been studied in the treatment of AR and allergic asthma, especially for the management of severe uncontrolled phenotypes. Among these, omalizumab, a humanized monoclonal antibody that binds circulating $\operatorname{IgE}$ and prevents its attachment to high-affinity IgE receptors, is available worldwide. Omalizumab improves both upper and lower airway diseases, reducing nasal and asthma symptoms, decreasing exacerbations, and improving quality of life. ${ }^{66}$ Mepolizumab, a monoclonal antibody that blocks the binding of IL-5 to eosinophils, has also shown a beneficial effect on severe eosinophilic airway diseases, such as asthma and nasal polyposis in adults. ${ }^{67-69}$ Because these treatments have systemic effects, it is not possible to design a study to assess how much the improvement in asthma is, due to direct effects or indirect effects associated with rhinitis improvement.

The management of rhinitis may promote better adherence to therapy. It should consider severity and duration of the disease and patient preference, as well as the efficacy, availability, and cost of medications. Therefore, management of rhinitis and asthma must be jointly carried out, including environmental control, pharmacotherapy, and SIT.

\section{Conclusion}

The treatment of rhinitis is indispensable in patients with asthma, since it leads to better control of both diseases, and the lessons of the ARIA initiative cannot be forgotten. Further studies regarding UAD are needed to better understand the interactions between the upper and lower airways, but there is no doubt that rhinitis and asthma have to be studied and managed in an integrated manner.

\section{Disclosure}

The authors report no conflicts of interest in this work. 


\section{References}

1. Bousquet J, van Cauwenberge P, Khaltaev N. Allergic rhinitis and its impact on asthma. J Allergy Clin Immunol. 2001;108(5 Suppl): S147-S334.

2. Cingi C, Muluk NB, Cobanoglu B, Çatli T, Dikici O. Nasobronchial interaction. World J Clin Cases. 2015;3(6):499-503.

3. YusufOM. Streptococcus pyogenes upper respiratory infections and their effect on atopic conditions. Prim Care Respir J. 2012;21(2):126-127.

4. Bousquet J, Khaltaev N, Cruz AA, et al. Allergic rhinitis and its impact on asthma (ARIA) 2008. Allergy. 2008;63(Suppl 86):8-160.

5. Kaliner M, McFadden F. Bronchial asthma. In: Samter S, editor. Immunological Diseases. 4th ed. Boston: Little Brown; 1988: 1067-1118.

6. Ciprandi G, Caimmi D, Miraglia Del Giudice M, La Rosa M, Salpietro C, Marseglia GL. Recent developments in united airways disease. Allergy Asthma Immunol Res. 2012;4(4):171-177.

7. Eccles C. Anatomy and physiology of the nose and control of nasal airflow. In: Adkinson NF, Yunginger JW, Busse WW, et al, editors. Middleton's Allergy: Principles and Practice. 7th ed. Philadelphia: Elsevier; 2008:701-711.

8. Seidman MD, Gurgel RK, Lin SY, et al. Clinical practice guideline: allergic rhinitis. Otolaryngol Head Neck Surg. 2015;152(Suppl 1): $\mathrm{S} 1-\mathrm{S} 43$.

9. Celedon JC, Palmer LJ, Weiss S, et al. Asthma, rhinitis, and skin test reactivity to aeroallergens in families of asthmatic subjects in Anqing, China. Am J Respir Crit Care Med. 2001;163(5):1108-1112.

10. Gaga M, Lambrou P, Papageorgiou N, et al. Eosinophils are a feature of upper and lower airway pathology in non-atopic asthma, irrespective of the presence of rhinitis. Clin Exp Allergy. 2000;30(5):663-669.

11. FitzGerald J, Bateman E, Boulet L, et al. Global strategy for asthma management and prevention. 2015. Available from: http://www.ginasthma.org/documents/4. Accessed June 17, 2015.

12. Lieberman P, Pattanaik D. Nonallergic rhinitis. Curr Allergy Asthma Rep. 2014;14(6):439.

13. Vanna AT, Yamada E, Arruda LK, Naspitz CK, Solé D. International Study of Asthma and Allergies in Childhood: validation of the rhinitis symptom questionnaire and prevalence of rhinitis in schoolchildren in São Paulo, Brazil. Pediatr Allergy Immunol. 2001;12(2):95-101.

14. Bisaccioni C, Aun MV, Cajuela E, Kalil J, Agondi RC, GiavinaBianchi P. Comorbidities in severe asthma: frequency of rhinitis, nasal polyposis, gastroesophageal reflux disease, vocal cord dysfunction and bronchiectasis. Clinics. 2009;64(8):769-773.

15. Crapo RO, Casaburi R, Coates AL, et al. Guidelines for methacholine and exercise challenge testing - 1999. Am J Respir Crit Care Med. 2000;161(1):309-329.

16. Madonini E, Briatico-Vangosa G, Pappacoda A, Maccagni G, Cardani A, Saporiti F. Seasonal increase of bronchial reactivity in allergic rhinitis. J Allergy Clin Immunol. 1987;79(2):358-363.

17. Shaaban R, Zureik M, Soussan D, et al. Rhinitis and onset of asthma: a longitudinal population-based study. Lancet. 2008;372(9643): 1049-1057.

18. Rochat MK, Illi S, Ege MJ, et al. Allergic rhinitis as a predictor for wheezing onset in school-aged children. J Allergy Clin Immunol. 2010;126(6):1170-1175.

19. Settipane RJ, Hagy GW, Settipane GA. Long-term risk-factors for developing asthma and allergic rhinitis: a 23-year follow-up study of college students. Allergy Proc. 1994;15(1):21-25.

20. Guerra S, Sherrill DL, Martinez FD, Barbee RA. Rhinitis as an independent risk factor for adult-onset asthma. J Allergy Clin Immunol. 2002;109(3):419-425.

21. Leynaert B, Bousquet J, Neukirch C, et al. Perennial rhinitis: an independent risk factor for asthma in nonatopic subjects - results from the European Community Respiratory Health Survey. J Allergy Clin Immunol. 1999;104(2 Pt 1):301-304.

22. Porsbjerg C, von Linstow ML, Ulrik CS, Nepper-Christensen S, Backer V. Risk factors for onset of asthma: a 12-year prospective follow-up study. Chest. 2006;129(2):309-316.
23. Feng CH, Miller MD, Simon RA. The united allergic airway: connections between allergic rhinitis, asthma, and chronic sinusitis. Am J Rhinol Allergy. 2012;26(3):187-190.

24. Okano M, Kariya S, Ohta N, Imoto Y, Fujieda S, Nishizaki K. Association and management of eosinophilic inflammation in upper and lower airways. Allergol Int. 2015;64(2):131-138.

25. Togias A. Mechanisms of nose-lung interaction. Allergy. 1999; 54(Suppl 57):94-105.

26. Lambrecht BN, Hammad H. The immunology of asthma. Nat Immunol. 2015;16(1):45-56.

27. Chawes BL, Bønnelykke K, Kreiner-Møller E, Bisgaard H. Children with allergic and nonallergic rhinitis have a similar risk of asthma. J Allergy Clin Immunol. 2010;126(3):567-573.

28. Stone KD, Prussin C, Metcalfe DD. IgE, mast cells, basophils, and eosinophils. J Allergy Clin Immunol. 2010;125(2 Suppl 2):S73-S80.

29. Rosenberg HF, Dyer KD, Foster PS. Eosinophils: changing perspectives in health and disease. Nat Rev Immunol. 2013;13(1):9-22.

30. de Magalhães Simões S, dos Santos MA, da Silva Oliveira M, et al. Inflammatory cell mapping of the respiratory tract in fatal asthma. Clin Exp Allergy. 2005;35(5):602-611.

31. Brugman SM, Larsen GL, Henson PM, Honor J, Irvin CG. Increased lower airways responsiveness associated with sinusitis in a rabbit model. Am Rev Respir Dis. 1993;147(2):314-320.

32. Chung KF, Adcock IM. Clinical phenotypes of asthma should link up with disease mechanisms. Curr Opin Allergy Clin Immunol. 2015;15(1):56-62.

33. Braunstahl GJ, Kleinjan A, Overbeek SE, Prins JB, Hoogsteden HC, Fokkens WJ. Segmental bronchial provocation induces nasal inflammation in allergic rhinitis patients. Am J Respir Crit Care Med. 2000;161(6):2051-2057.

34. Xie JX, Xi Y, Zhang Q, et al. An intratracheal challenge murine model of asthma: can bronchial inflammation affect the nose? Allergy Asthma Immunol Res. 2015;7(1):76-82.

35. Fontanari P, Burnet H, Zattara-Hartmann MC, Jammes Y. Changes in airway resistance induced by nasal inhalation of cold dry, dry, or moist air in normal individuals. J Appl Physiol (1985). 1996;81(4):1739-1743.

36. Fontanari P, Zattara-Hartmann MC, Burnet H, Jammes Y. Nasal eupnoeic inhalation of cold, dry air increases airway resistance in asthmatic patients. Eur Respir J. 1997;10(10):2250-2254.

37. Corren J, Adinoff AD, Irvin CG. Changes in bronchial responsiveness following nasal provocation with allergen. J Allergy Clin Immunol. 1992;89(2):611-618.

38. Baraniuk JN, Merck SJ. Nasal reflexes: implications for exercise, breathing, and sex. Curr Allergy Asthma Rep. 2008;8(2):147-153.

39. Gherson G, Moscato G, Vidi I, Salvaterra A, Candura F. Nonspecific nasal reactivity: a proposed method of study. Eur J Respir Dis. 1986; 69(1):24-28

40. Antonicelli L, Micucci C, Voltolini S, et al. Relationship between ARIA classification and drug treatment in allergic rhinitis and asthma. Allergy. 2007;62(9):1064-1070.

41. Halpern MT, Schmier JK, Richner R, Guo C, Togias A. Allergic rhinitis: a potential cause of increased asthma medication use, costs, and morbidity. J Asthma. 2004;41(1):117-126.

42. Greisner WA, 3rd Settipane RJ, Settipane GA. The course of asthma parallels that of allergic rhinitis: a 23-year follow-up study of college students. Allergy Asthma Proc. 2000;21(6):371-375.

43. Agondi RC, Machado ML, Kalil J, Giavina-Bianchi P. Intranasal corticosteroid administration reduces nonspecific bronchial hyperresponsiveness and improves asthma symptoms. $J$ Asthma. 2008;45(9):754-757.

44. Lohia S, Schlosser RJ, Soler ZM. Impact of intranasal corticosteroids on asthma outcomes in allergic rhinitis: a meta-analysis. Allergy. 2013;68(5):569-579.

45. Crystal-Peters J, Neslusan C, Crown WH, Torres A. Treating allergic rhinitis in patients with comorbid asthma: the risk of asthma-related hospitalizations and emergency department visits. J Allergy Clin Immunol. 2002;109(1):57-62. 
46. Stelmach R, do Patrocínio T Nunes M, Ribeiro M, Cukier A. Effect of treating allergic rhinitis with corticosteroids in patients with mild-tomoderate persistent asthma. Chest. 2005;128(5):3140-3147.

47. Taramarcaz P, Gibson PG. Intranasal corticosteroids for asthma control in people with coexisting asthma and rhinitis. Cochrane Database Syst Rev. 2003;(4):CD003570.

48. Giavina-Bianchi P, Aun MV, Bisaccioni C, Agondi R, Kalil J. Difficultto-control asthma management through the use of a specific protocol. Clinics (Sao Paulo). 2010;65(9):905-918.

49. Benninger M, Farrar JR, Blaiss M, et al. Evaluating approved medications to treat allergic rhinitis in the United States: an evidence-based review of efficacy for nasal symptoms by class. Ann Allergy Asthma Immunol. 2010;104(1):13-29.

50. Yamamoto H, Yonekura S, Sakurai D, et al. Comparison of nasal steroid with antihistamine in prophylactic treatment against pollinosis using an environmental challenge chamber. Allergy Asthma Proc. 2012;33(5):397-403.

51. Wilson AM, O’Byrne PM, Parameswaran K. Leukotriene receptor antagonists for allergic rhinitis: a systematic review and meta-analysis. Am J Med. 2004;116(5):338-344.

52. Nayak A, Langdon RB. Montelukast in the treatment of allergic rhinitis: an evidence-based review. Drugs. 2007;67(6):887-901.

53. Aubier M, Neukirch C, Peiffer C, Melac M. Effect of cetirizine on bronchial hyperresponsiveness in patients with seasonal allergic rhinitis and asthma. Allergy. 2001;56(1):35-42.

54. Zuberbier T, Bachert C, Bousquet PJ, et al. GA²LEN/EAACI pocket guide for allergen-specific immunotherapy for allergic rhinitis and asthma. Allergy. 2010;65(12):1525-1530.

55. Lin S, Erekosima N, Suarez-Cuervo C, et al. Allergen-Specific Immunotherapy for the Treatment of Allergic Rhinoconjunctivitis and/or Asthma: Comparative Effectiveness Review. Rockville (MD): Agency for Healthcare Research and Quality; 2013.

56. Wallace DV, Dykewicz MS, Bernstein DI, et al. The diagnosis and management of rhinitis: an updated practice parameter. J Allergy Clin Immunol. 2008;122(2 Suppl):S1-S84.

57. Niggemann B, Jacobsen L, Dreborg S, et al. Five-year follow-up on the PAT study: specific immunotherapy and long-term prevention of asthma in children. Allergy. 2006;61(7):855-859.
58. Möller C, Dreborg S, Ferdousi HA, et al. Pollen immunotherapy reduces the development of asthma in children with seasonal rhinoconjunctivitis (the PAT-Study). J Allergy Clin Immunol. 2002;109(2):251-256.

59. Jacobsen L, Niggemann B, Dreborg S, et al. Specific immunotherapy has long-term preventive effect of seasonal and perennial asthma: 10-year follow-up on the PAT study. Allergy. 2007;62(8):943-948.

60. Polosa R, Li Gotti F, Mangano G, et al. Effect of immunotherapy on asthma progression, BHR and sputum eosinophils in allergic rhinitis. Allergy. 2004;59(11):1224-1228.

61. Morjaria JB, Caruso M, Rosalia E, Russo C, Polosa R. Preventing progression of allergic rhinitis to asthma. Curr Allergy Asthma Rep. 2014;14(2):412.

62. Novembre E, Galli E, Landi F, et al. Coseasonal sublingual immunotherapy reduces the development of asthma in children with allergic rhinoconjunctivitis. J Allergy Clin Immunol. 2004;114(4):851-857.

63. Des Roches A, Paradis L, Menardo JL, Bouges S, Daurés JP, Bousquet J. Immunotherapy with a standardized Dermatophagoides pteronyssinus extract. VI. Specific immunotherapy prevents the onset of new sensitizations in children. J Allergy Clin Immunol. 1997;99(4):450-453.

64. Inal A, Altintas DU, Yilmaz M, Karakoc GB, Kendirli SG, SertdemirY. Prevention of new sensitizations by specific immunotherapy in children with rhinitis and/or asthma monosensitized to house dust mite. J Investig Allergol Clin Immunol. 2007;17(2):85-91.

65. Marogna M, Tomassetti D, Bernasconi A, et al. Preventive effects of sublingual immunotherapy in childhood: an open randomized controlled study. Ann Allergy Asthma Immunol. 2008;101(2):206-211.

66. Gevaert P, Calus L, Van Zele T, et al. Omalizumab is effective in allergic and nonallergic patients with nasal polyps and asthma. J Allergy Clin Immunol. 2013;131(1):110e1-116e1.

67. Robinson DS. Mepolizumab for severe eosinophilic asthma. Exp Rev Respir Med. 2013;7(1):13-17.

68. Pavord ID, Korn S, Howarth P, et al. Mepolizumab for severe eosinophilic asthma (DREAM): a multicentre, double-blind, placebocontrolled trial. Lancet. 2012;380(9842):651-659.

69. Gevaert P, Van Bruaene N, Cattaert T, et al. Mepolizumab, a humanized anti-IL-5 mAb, as a treatment option for severe nasal polyposis. J Allergy Clin Immunol. 2011;128(5):989-995.e1-e8.

\section{Publish your work in this journal}

The Journal of Asthma and Allergy is an international, peer-reviewed open-access journal publishing original research, reports, editorials and commentaries on the following topics: Asthma; Pulmonary physiology; Asthma related clinical health; Clinical immunology and the immunological basis of disease; Pharmacological interventions and

\section{Dovepress}

new therapies. Issues of patient safety and quality of care will also be considered. The manuscript management system is completely online and includes a very quick and fair peer-review system, which is all easy to use. Visit http://www.dovepress.com/testimonials.php to read real quotes from published authors. 